\title{
Article \\ Impact of Bundle Branch Block on Permanent Pacemaker Implantation after Transcatheter Aortic Valve Implantation: A Meta-Analysis
}

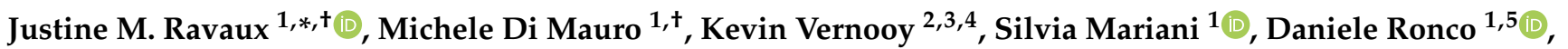 \\ Jorik Simons ${ }^{1}{ }^{(1)}$, Arnoud W. Van't Hof ${ }^{2,3}$, Leo Veenstra ${ }^{2}$, Suzanne Kats ${ }^{1}$, Jos G. Maessen ${ }^{1,3}$ and \\ Roberto Lorusso 1,3 (D)
}

Citation: Ravaux, J.M.; Mauro, M.D. Vernooy, K.; Mariani, S.; Ronco, D.; Simons, J.; Van't Hof, A.W.; Veenstra, L.; Kats, S.; Maessen, J.G.; et al. Impact of Bundle Branch Block on Permanent Pacemaker Implantation after Transcatheter Aortic Valve Implantation: A Meta-Analysis. J. Clin. Med. 2021, 10, 2719. https:// doi.org/10.3390/jcm10122719

Academic Editors: Michał Ciurzyński and Justyna Domienik-Karłowicz

Received: 3 May 2021

Accepted: 15 June 2021

Published: 19 June 2021

Publisher's Note: MDPI stays neutral with regard to jurisdictional claims in published maps and institutional affiliations.

Copyright: () 2021 by the authors Licensee MDPI, Basel, Switzerland. This article is an open access article distributed under the terms and conditions of the Creative Commons Attribution (CC BY) license (https:/ / creativecommons.org/licenses/by/ $4.0 /)$.
1 Department of Cardio-Thoracic Surgery, Heart and Vascular Centre, Maastricht University Medical Centre (MUMC), 6202 AZ Maastricht, The Netherlands; mdimauro1973@gmail.com (M.D.M.); s.mariani1985@gmail.com (S.M.); daniele.ronco@LIVE.IT (D.R.); Jorik_Simons@hotmail.com (J.S.); suzanne.kats@mumc.nl (S.K.); j.g.maessen@mumc.nl (J.G.M.); roberto.lorussobs@gmail.com (R.L.)

2 Department of Cardiology, Maastricht University Medical Centre (MUMC), 6202 AZ Maastricht, The Netherlands; kevin.vernooy@mumc.nl (K.V.); arnoud.vant.hof@mumc.nl (A.W.V.H.); l.veenstra@mumc.nl (L.V.)

3 Cardiovascular Research Institute Maastricht (CARIM), Maastricht University Medical Center, 6202 AZ Maastricht, The Netherlands

4 Department of Cardiology, Radboud University Medical Center (Radboudumc), 6525 GA Nijmegen, The Netherlands

5 Department of Medicine and Surgery, Circolo Hospital, University of Insubria, 21100 Varese, VA, Italy

* Correspondence: jmravaux@hotmail.com

+ Co-author, equally contributors.

Abstract: Data regarding the impact of infra-Hisian conduction disturbances leading to permanent pacemaker implantation (PPI) after transcatheter aortic valve implantation (TAVI) remain limited. The aim of this study was to determine the impact of right and/or left bundle branch block (RBBB/LBBB) on post-TAVI PPI. We performed a systematic literature review to identify studies reporting on RBBB and/or LBBB status and post-TAVI PPI. Study design, patient characteristics, and the presence of branch block were analyzed. Odds ratios (ORs) with 95\% CI were extracted. The final analysis included 36 studies, reporting about 55,851 patients. Data on LBBB were extracted from 33 studies. Among 51,026 patients included, 5503 showed pre-implant LBBB (11.9\% (10.4\%-13.8\%)). The influence of LBBB on post-TAVI PPI was not significant OR $1.1474(0.9025 ; 1.4588), p=0.2618$. Data on RBBB were extracted from 28 studies. Among 46,663 patients included, 31,603 showed pre-implant RBBB $(9.2 \%(7.3 \%-11.6 \%))$. The influence of RBBB on post-TAVI PPI was significant OR $4.8581(4.1571 ; 5.6775), p<0.0001$. From this meta-analysis, the presence of RBBB increased the risk for post-TAVI PPI, independent of age or LVEF, while this finding was not confirmed for patients experimenting with LBBB. This result emphasizes the need for pre-operative evaluation strategies in patient selection for TAVI.

Keywords: transcatheter aortic valve implantation; right bundle branch block; left bundle branch block; permanent pacemaker implantation

\section{Introduction}

Atrio-ventricular conduction disturbances and subsequent permanent pacemaker implantation (PPI) represent frequent complication after transcatheter aortic valve implantation (TAVI) [1,2]. Notably, infra-Hisian conductions' desynchrony such as left bundle branch block (LBBB) and right bundle branch block (RBBB) remain an ongoing issue in TAVI [3,4], especially considering TAVI as well-established therapeutic approach for patients with aortic stenosis at high surgical risk [5], while considerable advances in procedural techniques tend to extend TAVI indications to patients with a lower surgical risk [6]. 
Mechanical stress on the aortic valve annulus, deterioration of the ventricular septum, and local edema may all injury the atrio-ventricular conduction during the TAVI procedures $[1,3]$. In patients with pre-existing conduction system impairments, such additional procedural-related factors may contribute to a higher post-TAVI PPI rate. Consequently, complications such as PPI thus remain a substantial barrier to extending this technique to operable patients who would otherwise undergo surgery [7]. Therefore, better patient selection and identification of pre-operative risk factors for progression of conduction disturbances, and subsequently PPI, are decisive [8]. Current data about the clinical impact of bundle branch block on post-TAVI PPI remain controversial $[9,10]$. Left bundle branch block (LBBB) occurs in 5 to $65 \%$ in TAVI patients and leads to PPI in 15 of $20 \%$ of cases [11]. Pre-operative right bundle branch block (RBBB) is present in 10 to $21 \%$ of patients and results in up to $40 \%$ of post-operative PPI, making pre-operative RBBB the most important patient-related factor $[2,12]$. However, the prognostic value on pre-existing infra-Hisian conduction disturbances on post-TAVI PPI remains unclear [10-12].

We aim to investigate the clinical impact of pre-operative RBBB/LBBB on PPI after TAVI.

\section{Materials and Methods}

\subsection{Research Strategy}

This meta-analysis was performed in accordance with the Preferred Reporting Items for Systematic Reviews and Meta-Analyses (PRISMA) and the research strategy was developed according to available guidance from the Cochrane Collaboration. A broad, computerized literature search was performed to identify all relevant studies from Embase, Cochrane database, and PubMed exploring Medical Subject Heading (MeSH) terms related to pre-operative RBBB or pre-operative/new-onset LBBB in TAVI population. The PubMed database was searched entering the following key words: "Pacemaker, Artificial" [Mesh] OR pacemaker implantation AND "Transcatheter Aortic Valve Replacement" [Mesh] OR transcatheter aortic valve replacement AND "Bundle-Branch Block" [Mesh]. We restricted the research to English publications. Last access to the database was on 1 November 2020. The search was limited to studies in human.

\subsection{Eligibility Criteria and Studies Selection}

Studies were included in the final analyses if patients were $>18$ years (I); $>250$ patients were included in the main analysis, in order to provide data interpretation of the most consistent clinical series (II); and studies provided a description of pacemaker status of the population (III). Furthermore, articles with no possible extraction of the presence of RBBB/LBBB were excluded. Pre-operative RBBB/LBBB and new-onset RBBB/LBBB were both included in the present analysis. Systematic review and meta-analyses were not taken in account. Studies describing cardiac surgery procedures were also left out. The selected articles underwent extensive evaluation at title and abstract level by two independent researchers (J.R. and M.D.M.) to assess the potential inclusion in the metaanalysis. Discrepancies were solved by consensus with the intervention of a third reviewer (R.L.). There were no duplicate data.

\subsection{Statistical Analysis}

Calculation of an overall proportion from studies reporting a single proportion was performed using a meta-analytic approach by means of metaprop function of meta package in R. A logit-transformation was performed as suggested by Warton \& Hui [13] to calculate confidence intervals (CIs) for individual study results. A Clopper-Pearson approach and a DerSimonian-Laird estimator were used to estimate the between-study variance [14]. Total proportion with $95 \% \mathrm{Cl}$ was reported. Funnel plot and Egger's test were used for estimation of publication bias. The primary endpoint was 30-day or in-hospital PPI after TAVI, so odds ratios (OR) with $95 \%$ CI were extracted from 36 studies. Statistical pooling of OR was performed using a random effect model with 95\% CI. Forest plots were used 
to plot the effect size, either for each study or overall. We calculated the I2 statistics $(0 \% \sim 100 \%)$ to explain the between-study heterogeneity, with I2 $\leq 25 \%$ suggesting more homogeneity, $25 \%<\mathrm{I} 2 \leq 75 \%$ suggesting moderate heterogeneity, and $\mathrm{I} 2>75 \%$ suggesting high heterogeneity $[15,16]$. If the null hypothesis was rejected, a random effects model was used to calculate pooled effect estimations. If the null hypothesis was not rejected, a fixed effects model was used to calculate pooled effect estimations [14]; 95\% CI was also reported. Forest plots were used to plot the effect size, either for each study or overall. Publication bias was evaluated by graphical inspection of funnel plot; estimation of publication bias was quantified by means of Egger's linear regression test [17]. In the case of moderate or high heterogeneity, influence analysis was performed with different approaches: Baujat plot [18] and a leave-one-out sensitivity analysis were performed by iteratively removing one study at a time to confirm that our findings were not driven by any single study. Metaregression analysis was performed, reporting results as regression coefficient (i.e., Beta) and $p$-value. One removed analysis was performed as a sensitivity analysis. "Meta package" in R-studio version 1.1.463 (2009-2018) was used. Because this study was a systematic review and meta-analysis based on published articles, ethical approval was waived by the institutional review board of the University Hospital of Maastricht.

\section{Results}

\subsection{Study Inclusion}

Our search yielded 877 records initially screened at the title and abstract level, with 222 papers fully reviewed for eligibility. There were no duplicate data. Ultimately, 36 studies were identified and provided data for the research analysis (Supplementary Figure S1).

\subsection{Baseline Characteristics of Included Patients and Permanent Pacemaker Implantation Details}

Table 1 shows the baseline characteristics of the included studies. The 36 studies included a total of 55,851 patients in the final analysis, from 2005 to 2018 [19-54]. The mean age of the patients was 81.9 years, with a mean STS score of 8.3. Only five studies were prospective in nature $[35,40,41,44,51]$. The PPI details in the included studies are reported in Table 2. The overall incidence of PPI reached $15.2 \%$, ranging from $4.3 \%$ to $32 \%$. 
Table 1. Baseline characteristics of included studies $(n=36)$. TAVI, transcatheter aortic valve implantation.

\begin{tabular}{|c|c|c|c|c|c|c|c|c|c|c|}
\hline Study & Year & $\begin{array}{l}\text { Study Design } \\
\text { (Nr Centers) }\end{array}$ & $\begin{array}{l}\text { Sample } \\
\text { Size }\end{array}$ & $\begin{array}{c}\text { Age } \\
\text { (Years) }\end{array}$ & $\begin{array}{l}\text { STS-Score } \\
(\%)\end{array}$ & Inclusion Period & Valve Type & $\begin{array}{l}\text { Follow-Up } \\
\text { (Months) }\end{array}$ & $\begin{array}{l}\text { Approach for } \\
\text { TAVI }\end{array}$ & $\begin{array}{c}\text { Mortality at } \\
30 \text { Days }\end{array}$ \\
\hline De Carlo et al. [19] & 2012 & Retrospective (3) & 275 & 82.4 & na & Sep 2007-Jul 20120 & $100 \% \mathrm{MCV}$ & 12 & na & $3 \%$ \\
\hline Gensas et al. [20] & 2014 & Retrospective (18) & 353 & 82 & 14.4 & Jan 2008-Feb 2012 & $\begin{array}{l}85.8 \% \mathrm{SE} \\
14.2 \% \mathrm{BE}\end{array}$ & 60 & na & na \\
\hline Mouillet et al. [21] & 2015 & Retrospective (29) & 833 & 82 & 14.1 & Jan 2010-Oct 2011 & $100 \% \mathrm{SE}$ & 8 & na & $9.3 \%$ \\
\hline Nazif et al. [22] & 2015 & Retrospective (21) & 1973 & 84.5 & 11.4 & May 2007-Sept 2011 & $100 \% \mathrm{BE}$ & 12 & na & $6.6 \%$ \\
\hline Rodriguez-Olivares et al. [23] & 2016 & Retrospective (1) & 302 & 81 & na & Nov 2005-Jan 2015 & $\begin{array}{c}67.2 \% \mathrm{SE} \\
21.2 \% \mathrm{BE} \\
11.6 \% \mathrm{ME}\end{array}$ & na & na & na \\
\hline Gonska et al. [24] & 2017 & Retrospective (1) & 283 & 79.9 & 6.7 & na & $100 \%$ ES3 & na & na & na \\
\hline Raelson et al. [25] & 2017 & Retrospective (1) & 578 & 85,5 & $\mathrm{Na}$ & Mar 2009-Dec 2014 & $\begin{array}{l}21 \% \mathrm{SE} \\
79 \% \mathrm{BE}\end{array}$ & 1 & na & na \\
\hline Dumonteil et al. [26] & 2017 & Retrospective (14) & 250 & 84 & 6,3 & Oct 2012-May 2014 & $100 \% \mathrm{ME}$ & 12 & $100 \% \mathrm{TF}$ & $4 \%$ \\
\hline Monteiro et al. [27] & 2017 & Retrospective (22) & 670 & 81.8 & 10.7 & Jan 2008-Jan 2015 & $\begin{array}{c}74 \% \mathrm{MCV} \\
26 \% \mathrm{ES}\end{array}$ & na & $\begin{array}{c}96 \% \text { TF } \\
4 \% \text { others }\end{array}$ & na \\
\hline Pellegrini et al. [28] & 2018 & Retrospective (3) & 283 & 80.8 & 6 & Jan 2014-Jan 2016 & $100 \% \mathrm{SE}$ & 12 & $100 \% \mathrm{TF}$ & na \\
\hline Mangieri et al. [30] & 2018 & Retrospective (1) & 611 & 84.4 & 6.9 & Oct 2007-Jul 2015 & $\begin{array}{l}51.7 \% \mathrm{BE} \\
33.7 \% \mathrm{SE}\end{array}$ & 12 & na & na \\
\hline Bhardwaj et al. [31] & 2018 & Retrospective (1) & 383 & 83 & 9 & Jan 2012-July 2016 & $\begin{array}{l}82 \% \mathrm{BE} \\
18 \% \mathrm{SE}\end{array}$ & 9 & $84 \% \mathrm{TF}$ & na \\
\hline Pellegrini et al. [32] & 2018 & Retrospective (3) & 709 & 81 & na & Jan 2014-Jan 2016 & $100 \% \mathrm{BE}$ & na & $100 \% \mathrm{TF}$ & $1.6 \%$ \\
\hline Gaede et al. [33] & 2018 & Retrospective (1) & 1025 & 81.9 & na & 2010-2015 & na & 2.4 & na & na \\
\hline Nadeem et al. [34] & 2018 & Retrospective (1) & 672 & 81.4 & 7.4 & 2011-2017 & na & 12 & na & na \\
\hline Chamandi et al. [35] & 2018 & Prospective (9) & 1629 & 81.5 & 10.9 & May 2009-Feb 2015 & $\begin{array}{l}50.3 \% \mathrm{BE} \\
49.7 \% \mathrm{SE}\end{array}$ & 48 & na & $42.3 \%$ \\
\hline Doshi et al. [36] & 2018 & Retrospective (na) & 8148 & 82.5 & na & Jan 2012-Dec 2014 & na & na & na & na \\
\hline
\end{tabular}


Table 1. Cont.

\begin{tabular}{|c|c|c|c|c|c|c|c|c|c|c|}
\hline Study & Year & $\begin{array}{l}\text { Study Design } \\
\text { (Nr Centers) }\end{array}$ & $\begin{array}{l}\text { Sample } \\
\text { Size }\end{array}$ & $\begin{array}{c}\text { Age } \\
\text { (Years) }\end{array}$ & $\begin{array}{c}\text { STS-Score } \\
(\%)\end{array}$ & Inclusion Period & Valve Type & $\begin{array}{l}\text { Follow-Up } \\
\text { (Months) * }\end{array}$ & $\begin{array}{c}\text { Approach for } \\
\text { TAVI }\end{array}$ & $\begin{array}{l}\text { Mortality at } \\
30 \text { Days }\end{array}$ \\
\hline Vejpongsa et al. [37] & 2018 & Retrospective (na) & 18,400 & 81.2 & na & Jan 2012-Dec 2013 & na & na & $\begin{array}{l}\text { TF } 75.5 \% \\
24.5 \% \text { TA }\end{array}$ & na \\
\hline Dolci et al. [39] & 2019 & Retrospective (1) & 266 & 80 & na & Feb 2014-Feb 2018 & $100 \% \mathrm{BE}$ & 12 & $\begin{array}{l}84 \% \mathrm{TF} \\
16 \% \mathrm{TA}\end{array}$ & na \\
\hline Costa et al. [40] & 2019 & Prospective (1) & 1116 & 82 & 4.4 & June 2007-Feb 2018 & $\begin{array}{c}\text { 61.8\% SE } \\
27.2 \% \mathrm{BE} \\
0.5 \% \mathrm{ME} \\
10.5 \% \text { Others }\end{array}$ & 72 & $\begin{array}{c}97 \% \mathrm{TF} \\
3 \% \text { others }\end{array}$ & $3.9 \%$ \\
\hline Meduri et al. [41] & 2019 & Prospective (1) & 704 & 82.5 & 6.6 & na & $\begin{array}{l}34 \% \mathrm{SE} \\
66 \% \mathrm{ME}\end{array}$ & 12 & na & na \\
\hline Du et al. [42] & 2020 & Retrospective (1) & 256 & 76.5 & 7.1 & Mar 2013-Oct 2018 & na & 12 & $\mathrm{Na}$ & $3.3 \%$ \\
\hline Shivamurthy et al. [43] & 2020 & Retrospective (1) & 917 & 80 & na & Nov 2011-Feb 2017 & na & na & $\begin{array}{l}89.7 \% \text { TF } \\
10.3 \% \text { TA }\end{array}$ & na \\
\hline \multicolumn{11}{|c|}{ Studies reporting only on RBBB status in pacemaker population $(n=3)$} \\
\hline Watanabe et al. [44] & 2016 & Prospective (9) & 749 & 85 & 6.9 & Oct 2013-Aug 2015 & $100 \% \mathrm{BE}$ & 16.4 & $\begin{array}{c}78.5 \% \text { TF } \\
18.8 \% \text { TA } \\
2.7 \% \text { T iliac }\end{array}$ & $4 \%$ \\
\hline Auffret et al. [45] & 2017 & Retrospective (na) & 3527 & 82 & na & na & $\begin{array}{l}55.8 \% \mathrm{BE} \\
44.2 \% \mathrm{SE}\end{array}$ & 23 & $\begin{array}{c}79.6 \% \mathrm{TF} \\
16.4 \% \mathrm{TA} \\
1.9 \% \mathrm{~T} \text { aortic } \\
2.1 \% \mathrm{TS}\end{array}$ & $7.2 \%$ \\
\hline Maeno et al. [46] & 2019 & Retrospective (1) & 659 & 83 & na & Jan 2013-Dec 2015 & $\begin{array}{l}85 \% \mathrm{BE} \\
15 \% \mathrm{SE}\end{array}$ & 19.1 & na & $2.6 \%$ \\
\hline \multicolumn{11}{|c|}{ Studies reporting only on LBBB status in pacemaker population $(n=8)$} \\
\hline Testa et al. [47] & 2013 & Retrospective (na) & 818 & 82 & na & Oct 2007-Apr 2011 & $100 \% \mathrm{SE}$ & 9 & $\begin{array}{l}88.6 \% \text { TF } \\
11.4 \% \text { TS }\end{array}$ & $5.5 \%$ \\
\hline Schymik et al. [48] & 2014 & Retrospective (1) & 624 & 82 & na & May 2008-Apr 2012 & $\begin{array}{l}80.8 \% \mathrm{BE} \\
19.2 \% \mathrm{SE}\end{array}$ & 12 & na & na \\
\hline Urena et al. [49] & 2014 & Retrospective (4) & 668 & 79.5 & na & na & $100 \% \mathrm{BE}$ & 13 & $\begin{array}{c}2.8 \% \text { TAortic } \\
42.9 \% \text { TA } \\
54.3 \% \text { TF }\end{array}$ & na \\
\hline
\end{tabular}


Table 1. Cont.

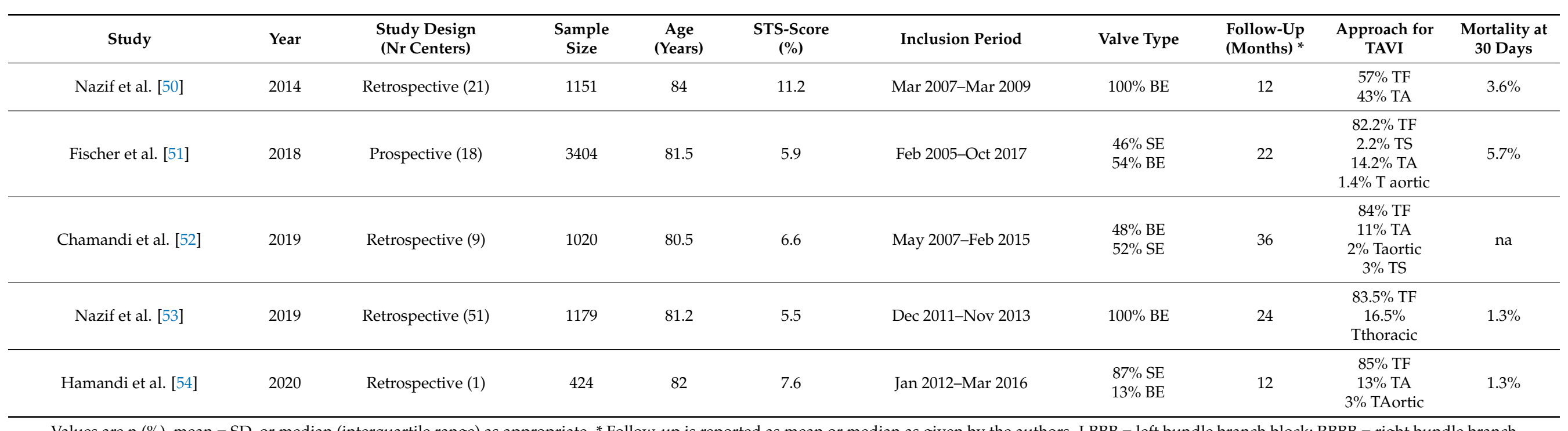

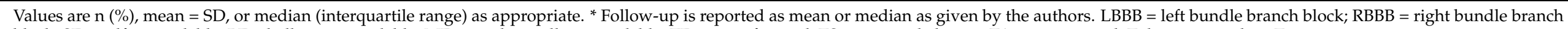
block; $\mathrm{SE}=$ self-expandable; $\mathrm{BE}=$ balloon-expandable; $\mathrm{ME}=$ mechanically-expandable; $\mathrm{TF}=$ trans-femoral; $\mathrm{TS}=$ trans-subclavian; $\mathrm{TA}=$ trans-apical; $\mathrm{T}$ iliac = trans-iliac; $\mathrm{T}$ aortic = trans-aortic. 
Table 2. Pacemaker-related details in included studies. PPI, pacemaker implantation.

\begin{tabular}{|c|c|c|c|c|c|c|c|c|c|}
\hline \multicolumn{10}{|c|}{ Studies Reporting on RBBB and LBBB Status in Pacemaker Population $(n=25)$} \\
\hline De Carlo et al. [19] & $\begin{array}{c}70 \% \text { AVB } \\
3 \% \text { SSS } \\
27 \% \text { others }\end{array}$ & 4 & $24 \%$ & 32 & 37 & 15 & 9 & $\begin{array}{c}\text { * lower MCV implantation } \\
\text { below aortic annulus } \\
* \text { RBBB } \\
* \text { left anterior hemiblock } \\
* \text { longer PR interval }\end{array}$ & na \\
\hline Gensas et al. [20] & na & na & $25.2 \%$ & 41 & 50 & 22 & 10 & $\begin{array}{l}* \text { pre-existing RBBB } \\
* \text { balloon pre-dilatation } \\
* \text { CoreValve use }\end{array}$ & na \\
\hline Mouillet et al. [21] & na & na & $30.3 \%$ & 115 & 106 & 60 & 24 & na & na \\
\hline Nazif et al. [22] & $\begin{array}{l}79 \% \text { AVB } \\
17.3 \% \text { SSS }\end{array}$ & 3 & $8.8 \%$ & 312 & 174 & 82 & 12 & $\begin{array}{l}\text { * Pre-existing RBBB } \\
\text { * Prosthesis to LV outflow } \\
\text { tract diameter ratio } \\
* \text { LV-end diastolic diameter }\end{array}$ & $\begin{array}{l}\text { * longer duration of hospitalization } \\
{ }^{*} \text { higher rates of repeat } \\
\text { hospitalization and mortality or } \\
\text { repeat hospitalization at } 1 \text { year }\end{array}$ \\
\hline Rodriguez-Olivares et al. [23] & na & na & $22.5 \%$ & 28 & 32 & 13 & 5 & na & $\begin{array}{c}{ }^{*} \text { more LVOT oversizing associated } \\
\text { with higher PPI }\end{array}$ \\
\hline Gonska et al. [24] & $\begin{array}{l}94.2 \% \text { AVB } \\
3.8 \% \text { B } \\
2 \% \text { others }\end{array}$ & 4,3 & $18.4 \%$ & 22 & 74 & 13 & 6 & $\begin{array}{c}* \text { baseline AV1B } \\
\text { * preprocedural complete } \\
\text { RBBB }\end{array}$ & na \\
\hline Raelson et al. [25] & $82 \%$ AVB & 3 & $9 \%$ & 65 & 50 & 19 & 3 & na & na \\
\hline Dumonteil et al. [26] & $\begin{array}{l}88.9 \% \text { AVB } \\
5.9 \% \text { others }\end{array}$ & 3 & $32 \%$ & 26 & 145 & 20 & 14 & $\begin{array}{c}{ }^{*} \text { baseline RBBB } \\
{ }^{*} \mathrm{LV} \text { outflow tract overstretch } \\
>10 \%\end{array}$ & $\begin{array}{c}\text { * trend lower PPI rate at } 30 \text { days } \\
\text { with shallower }(<=5 \mathrm{~mm}) \text { implant } \\
\text { depth }\end{array}$ \\
\hline Monteiro et al. [27] & na & na & $20.1 \%$ & 71 & 93 & 36 & 15 & $\begin{array}{c}* \text { previous RBBB } \\
* \text { mean aortic gradient } \\
>50 \mathrm{mmHg} \\
{ }^{*} \mathrm{MCV}\end{array}$ & na \\
\hline
\end{tabular}


Table 2. Cont

\begin{tabular}{|c|c|c|c|c|c|c|c|c|c|}
\hline Pellegrini et al. [28] & $\begin{array}{l}71.5 \% \text { AVB } \\
3.5 \% \text { SSS } \\
25 \% \text { B }\end{array}$ & na & $10 \%$ & 22 & 25 & 6 & 4 & * higher EuroSCORE & na \\
\hline De-Torres-Alba et al. [29] & $\begin{array}{c}96 \% \text { AVB } \\
1.4 \% \text { B } \\
2.6 \% \text { others }\end{array}$ & na & $12.5 \%$ & 20 & 74 & 7 & 11 & na & na \\
\hline Mangieri et al. [30] & $\begin{array}{c}84 \% \text { AVB } \\
8.4 \% \text { B }\end{array}$ & 0,3 & $8.8 \%$ & 37 & 61 & 7 & 5 & na & na \\
\hline Bhardwaj et al. [31] & na & na & $11.5 \%$ & 50 & 39 & 11 & 3 & $\begin{array}{l}\text { *PPI with short-term } \\
\text { reduction in QoL without } \\
\text { long-term implications }\end{array}$ & na \\
\hline Pellegrini et al. [32] & $\begin{array}{c}71.3 \% \text { AVB } \\
5.2 \% \text { SSS } \\
23.5 \% \text { B }\end{array}$ & na & $16.2 \%$ & 63 & 41 & 30 & 4 & $\begin{array}{c}* \text { increase in prosthesis } \\
\text { oversizing }\end{array}$ & na \\
\hline Gaede et al. [33] & $\begin{array}{l}90 \% \text { AVB } \\
8 \% \text { SSS } \\
2 \% \text { B }\end{array}$ & 4 & $14.7 \%$ & 98 & 107 & 31 & 17 & $\begin{array}{l}* \text { pre-existing RBBB } \\
\text { * CoreValve prosthesis }\end{array}$ & $\begin{array}{l}\text { Predictors of lack of recovery of } \\
\text { AVB } \\
\text { * prior RBBB } \\
\text { * higher mean aortic valve gradient } \\
{ }^{*} \text { post-dilatation of the prosthesis }\end{array}$ \\
\hline Nadeem et al. [34] & na & na & $21.7 \%$ & 113 & 51 & 65 & 4 & na & $\begin{array}{l}\text { * PPI more likely to have heart } \\
\text { failure admissions } \\
\text { * PPI trend toward increased } \\
\text { mortality }\end{array}$ \\
\hline Chamandi et al. [35] & $\begin{array}{l}76.7 \% \text { AVB } \\
5.6 \% \text { SSS } \\
3.1 \% \text { B } \\
14.6 \% \text { others }\end{array}$ & 2 & $19.8 \%$ & 169 & 179 & 84 & 37 & na & $\begin{array}{c}\text { * PPI higher rates of } \\
\text { rehospitalization due to heart } \\
\text { failure and combined endpoint of } \\
\text { mortality or heart failure } \\
\text { rehospitalization } \\
\text { * PPI lesser improvement in LVEF } \\
\text { over time, particularly in patients } \\
\text { with reduced LVEF before TAVI }\end{array}$ \\
\hline Doshi et al. [36] & na & na & $24 \%$ & 220 & 724 & 96 & 253 & $\begin{array}{c}\text { * female sex } \\
{ }^{*} \text { AF } \\
\text { * LBBB } \\
\quad \text { *AVB }\end{array}$ & na \\
\hline
\end{tabular}


Table 2. Cont.

\begin{tabular}{|c|c|c|c|c|c|c|c|c|c|}
\hline Vejpongsa et al. [37] & na & 2 & $9.9 \%$ & 715 & 1670 & 265 & 390 & na & na \\
\hline Cresse et al. [38] & na & 4 & $6.7 \%$ & 14 & 97 & 6 & 12 & na & $\begin{array}{l}* \text { RBBB, LBBB, } \triangle \mathrm{PR}>40 \mathrm{~ms} \\
\text { associated with PPI }\end{array}$ \\
\hline Dolci et al. [39] & $\begin{array}{l}80 \% \text { AVB } \\
11 \% \text { B } \\
9 \% \text { others }\end{array}$ & 4 & $13 \%$ & 29 & 41 & 12 & 3 & $\begin{array}{c}{ }^{*} \text { baseline RBBB } \\
{ }^{*} \text { QRS width immediately } \\
\text { after TAVI }\end{array}$ & na \\
\hline Costa et al. [40] & $\begin{array}{c}84.8 \% \text { AVB } \\
4.1 \% \text { SSS } \\
11 \% \text { Others }\end{array}$ & na & $13 \%$ & 92 & 99 & 39 & 8 & na & $\begin{array}{l}\text { * PPI associated with increased } 6 \\
\text { years mortality } \\
\text { * baseline RBBB higher chance of } \\
\text { being dependent at follow-up }\end{array}$ \\
\hline Meduri et al. [41] & $\begin{array}{l}90 \% \text { AVB } \\
6 \% \text { B } \\
4 \% \text { others }\end{array}$ & 2 & $28.4 \%$ & 85 & 56 & 68 & 20 & $\begin{array}{l}\text { * baseline RBBB } \\
\text { * mean depth of valve } \\
\text { implantation }\end{array}$ & $\begin{array}{l}{ }^{*} \text { medically-treated diabetes } \\
\text { mellitus in LOTUS valve patients }\end{array}$ \\
\hline Du et al. [42] & $89.5 \%$ AVB & 8.7 & $14.8 \%$ & 20 & 19 & 6 & 3 & na & na \\
\hline Shivamurthy et al. [43] & na & na & $9.8 \%$ & 130 & 79 & 38 & 9 & na & na \\
\hline \multicolumn{10}{|c|}{ Studies reporting only on RBBB status in pacemaker population $(n=3)$} \\
\hline Auffret et al. [45] & na & na & $16.5 \%$ & 362 & na & 137 & na & na & na \\
\hline Maeno et al. [46] & $\begin{array}{c}77.9 \% \text { AVB } \\
11.5 \% \text { SSS } \\
10.6 \%\end{array}$ & na & $15.8 \%$ & 101 & na & 38 & na & na & na \\
\hline \multicolumn{10}{|c|}{ Studies reporting only on LBBB status in pacemaker population $(n=8)$} \\
\hline Testa et al. [47] & na & na & $17.4 \%$ & na & 224 & na & 41 & na & $\begin{array}{l}\text { * LBBB associated with higher } \\
\text { short-term PPI }\end{array}$ \\
\hline Schymik et al. [48] & na & na & $10.8 \%$ & na & 197 & na & 28 & $\begin{array}{c}\text { * chronic AF } \\
\text { * baseline RBBB } \\
{ }^{*} \mathrm{MCV}\end{array}$ & na \\
\hline
\end{tabular}


Table 2. Cont.

\begin{tabular}{|c|c|c|c|c|c|c|c|c|c|}
\hline Urena et al. [49] & $\begin{array}{c}55.5 \% \text { AVB } \\
\text { SSS } 20.7 \% \\
24.1 \% \text { B }\end{array}$ & 365 & $4.3 \%$ & na & 128 & na & 11 & na & na \\
\hline Nazif et al. [50] & na & na & $5.1 \%$ & na & 121 & na & 12 & na & $\begin{array}{c}{ }^{*} \text { LBBB with higher PPI and failure } \\
\text { of LVEF }\end{array}$ \\
\hline Fischer et al. [51] & na & na & $15.5 \%$ & na & 398 & na & 84 & $\begin{array}{l}\text { * pre-existing LBBB increase } \\
\text { risk of death but not late PPI }\end{array}$ & $\begin{array}{l}\text { * pre-existing LBBB associated } \\
\text { with lower pre-operative LVEF }\end{array}$ \\
\hline Chamandi et al. [52] & na & na & $7.2 \%$ & na & 212 & na & 29 & na & na \\
\hline Hamandi et al. [54] & na & na & $18 \%$ & na & 52 & na & 6 & na & na \\
\hline
\end{tabular}

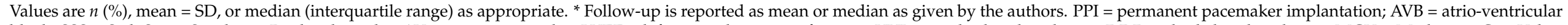

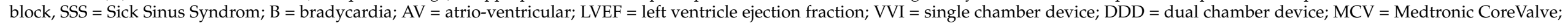

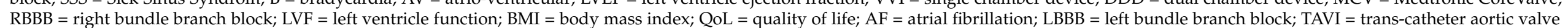
implantation; na = not available. 


\subsection{Influence of $\angle B B B$ on PPI}

Data on LBBB were extracted from 33 studies [19-43,47-54]; among the 51,026 patients included in the analysis, there were 5503 showing pre-implant LBBB. The cumulative proportion of LBBB was $11.9 \%(10.4 \%-13.8 \%)$ (Supplementary Figure S2). Heterogeneity was high $\left(\mathrm{I}^{2} 96.4 \%[95.6 \% ; 97.0 \%]\right)$. No publication bias was found $(p=0.2921)$. The cumulative proportion of LBBB in a subset of 7315 patients with post-TAVI PPI was 13.0\% $(10.6 \%-15.8 \%)$ with high heterogeneity $\left(\mathrm{I}^{2} 87.7 \%\right.$ [83.7\%; $\left.\left.90.6 \%\right]\right)$ and no publication bias $(p=0.3856)$ (Supplementary Figure S3). The cumulative proportion of LBBB in a subset of 43,650 patients without post-TAVI PPI was $12.3 \%(10.5 \%-14.4 \%)$ with high heterogeneity $\left(\mathrm{I}^{2} 96.5 \%\right.$ [95.8\%; 97.1\%]) and no publication bias $(p=0.6200)$ (Supplementary Figure S4).

The influence of LBBB on post-TAVI PPI was not significant OR $1.1474(0.9025 ; 1.4588$, $p=0.2618)$ with high heterogeneity $\mathrm{I}^{2}=86.2 \%[81.7 \% ; 89.6 \%]$ and no publication bias $(p=0.7100)$ (Figure 1). The baujat plot (Supplementary Figure S5) shows that the study by Vejpongsa et al. [37] may impact high heterogeneity, even if the sensitivity analysis does not confirm this hypothesis, as no influence of LBBB on post-TAVI PPI rate was evidenced at the leave-one out analysis (Figure 2). Meta-regression failed to identify some modifiers: age $(\mathrm{r}=-0.0592, p=0.4292)$, left ventricular ejection fraction (LVEF, $\mathrm{r}=0.0754, p=0.1741)$, and year of the study $(\mathrm{r}=-0.0008, p=0.9893)$ did not show any influence on the meta-analytic results (Supplementary Figures S6-S8).

\begin{tabular}{|c|c|c|c|c|c|c|}
\hline \multirow[b]{2}{*}{ Study } & \multicolumn{2}{|c|}{ Experimental } & \multicolumn{2}{|c|}{ Control } & \multicolumn{2}{|r|}{ Odds Ratio } \\
\hline & Events & Total & Events & Total & Weight & IV, Random, $95 \% \mathrm{CI}$ \\
\hline De Carlo 2012 & 9 & 37 & 57 & 238 & $2.9 \%$ & $1.052[0.477 ; 2.323]$ \\
\hline Testa 2013 & 41 & 224 & 101 & 594 & $3.8 \%$ & $1.100[0.738 ; 1.639]$ \\
\hline Gensas 2014 & 10 & 50 & 79 & 303 & $3.1 \%$ & $0.732[0.354 ; 1.512]$ \\
\hline Schymik 2014 & 28 & 197 & 41 & 437 & $3.6 \%$ & $1.606[0.965 ; 2.674]$ \\
\hline Ure 2014 & 11 & 128 & 18 & 540 & $3.0 \%$ & $2.764[1.289 ; 5.927]$ \\
\hline Zif 2014 & 12 & 121 & 30 & 1030 & $3.2 \%$ & $3.745[1.884 ; 7.445]$ \\
\hline Mouillet 2015 & 24 & 106 & 228 & 727 & $3.7 \%$ & $0.649[0.403 ; 1.046]$ \\
\hline Zif 2015 & 12 & 174 & 161 & 1799 & $3.4 \%$ & $0.780[0.429 ; 1.419]$ \\
\hline Rodriguez-Olivares 2016 & 5 & 32 & 63 & 270 & $2.6 \%$ & $0.654[0.251 ; 1.703]$ \\
\hline Dumonteuil 2017 & 1 & 14 & 80 & 236 & $1.4 \%$ & $0.216[0.039 ; 1.192]$ \\
\hline Gonska 2017 & 6 & 74 & 46 & 309 & $2.8 \%$ & $0.538[0.227 ; 1.274]$ \\
\hline Monteiro 2017 & 15 & 93 & 120 & 577 & $3.4 \%$ & $0.750[0.420 ; 1.340]$ \\
\hline Raelson 2017 & 3 & 50 & 51 & 528 & $2.2 \%$ & $0.683[0.222 ; 2.100]$ \\
\hline Bhardwaj 2018 & 3 & 39 & 41 & 344 & $2.2 \%$ & $0.701[0.223 ; 2.201]$ \\
\hline Chamandi 2018 & 37 & 179 & 285 & 1450 & $3.9 \%$ & $1.074[0.733 ; 1.575]$ \\
\hline De-Torres-Alba 2018 & 11 & 74 & 65 & 532 & $3.2 \%$ & $1.293[0.655 ; 2.550]$ \\
\hline Deem 2018 & 4 & 51 & 142 & 621 & $2.5 \%$ & $0.319[0.119 ; 0.853]$ \\
\hline Doshi 2018 & 253 & 724 & 1696 & 7424 & $4.2 \%$ & $1.815[1.544 ; 2.135]$ \\
\hline Fischer 2018 & 84 & 398 & 445 & 3006 & $4.1 \%$ & $1.545[1.191 ; 2.004]$ \\
\hline Gaede 2018 & 17 & 107 & 159 & 918 & $3.5 \%$ & $0.921[0.537 ; 1.579]$ \\
\hline Mangieri 2018 & 5 & 61 & 49 & 550 & $2.6 \%$ & $0.986[0.392 ; 2.482]$ \\
\hline Pellegrini 2018 & 4 & 41 & 111 & 668 & $2.5 \%$ & $0.600[0.221 ; 1.629]$ \\
\hline Pellegrini 2018 & 4 & 25 & 24 & 258 & $2.3 \%$ & $2.003[0.668 ; 6.006]$ \\
\hline Vejpongsa 2018 & 390 & 1670 & 1435 & 16730 & $4.2 \%$ & $3.249[2.866 ; 3.684]$ \\
\hline Chamandi 2019 & 29 & 212 & 44 & 808 & $3.6 \%$ & $2.762[1.688 ; 4.519]$ \\
\hline Costa 2019 & 8 & 99 & 137 & 1017 & $3.1 \%$ & $0.595[0.288 ; 1.229]$ \\
\hline Cresse 2019 & 12 & 97 & 14 & 289 & $2.9 \%$ & $2.778[1.255 ; 6.148]$ \\
\hline Dolci 2019 & 3 & 41 & 32 & 225 & $2.2 \%$ & $0.541[0.170 ; 1.719]$ \\
\hline Meduri 2019 & 20 & 56 & 225 & 648 & $3.5 \%$ & $1.055[0.600 ; 1.855]$ \\
\hline Zif 2019 & 9 & 179 & 9 & 1000 & $2.6 \%$ & $5.815[2.332 ; 14.503]$ \\
\hline Du 2020 & 3 & 19 & 35 & 237 & $2.1 \%$ & $1.210[0.362 ; 4.047]$ \\
\hline Hamandi 2020 & 6 & 52 & 84 & 372 & $2.8 \%$ & $0.477[0.203 ; 1.123]$ \\
\hline Shivamurthy 2020 & 9 & 79 & 81 & 838 & $3.1 \%$ & $1.252[0.613 ; 2.560]$ \\
\hline Total $(95 \% \mathrm{Cl})$ & & 5503 & & 45523 & $100.0 \%$ & $1.147[0.902 ; 1.459]$ \\
\hline Heterogeneity: Tau & & 231.99, & & $P<0.01)$ & $1^{2}=86 \%$ & \\
\hline
\end{tabular}

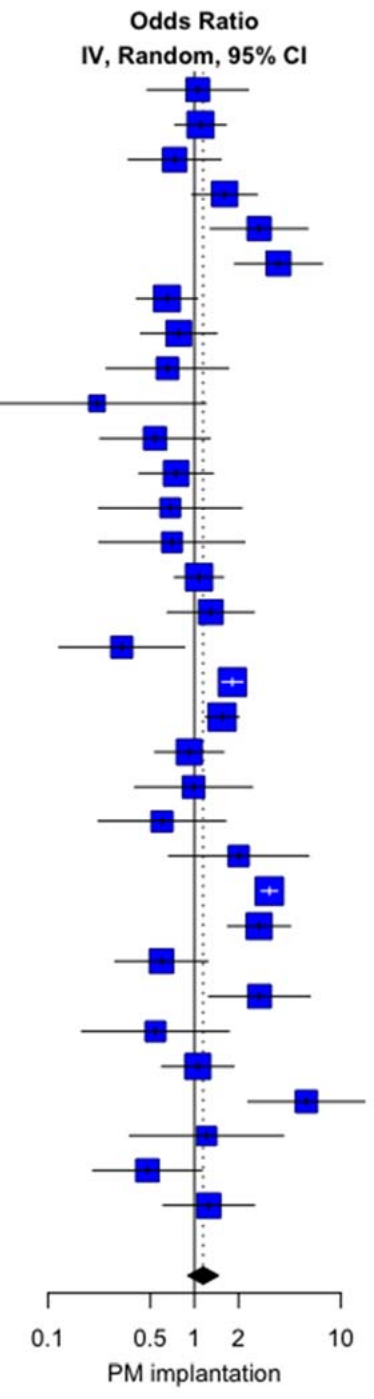

Figure 1. Forest plot comparing the effect of LBBB on the rate of post-TAVI PPI. IV= interval variable; $95 \%$ $\mathrm{CI}=$ confidence interval. 


Study
Omitting Bhardwaj 2018
Omitting Chamandi 2018
Omitting Chamandi 2019
Omitting Costa 2019
Omitting Cresse 2019
Omitting De Carlo 2012
Omitting De-Torres-Alba 2018
Omitting Deem 2018
Omitting Dolci 2019
Omitting Doshi 2018
Omitting Du 2020
Omitting Dumonteuil 2017
Omitting Fischer 2018
Omitting Gaede 2018
Omitting Gensas 2014
Omitting Gonska 2017
Omitting Hamandi 2020
Omitting Mangieri 2018
Omitting Meduri 2019
Omitting Monteiro 2017
Omitting Mouillet 2015
Omitting Pellegrini 2018
Omitting Pellegrini 2018
Omitting Raelson 2017
Omitting Rodriguez-Olivares 2016
Omitting Schymik 2014
Omitting Shivamurthy 2020
Omitting Testa 2013
Omitting Ure 2014
Omitting Vejpongsa 2018
Omitting Zif 2014
Omitting Zif 2015
Omitting Zif 2019

Total $(95 \% \mathrm{Cl})$
Odds Ratio

IV, Random, 95\% CI

$1.16[0.91 ; 1.48]$

$1.15[0.90 ; 1.47]$

$1.11[0.87 ; 1.42]$

$1.17[0.92 ; 1.49]$

$1.12[0.87 ; 1.43]$

$1.15[0.90 ; 1.47]$

$1.14[0.89 ; 1.46]$

$1.19[0.94 ; 1.51]$

$1.17[0.92 ; 1.49]$

$1.11[0.85 ; 1.46]$

$1.15[0.90 ; 1.46]$

$1.17[0.92 ; 1.49]$

$1.13[0.87 ; 1.46]$

$1.16[0.91 ; 1.48]$

$1.16[0.91 ; 1.48]$

$1.17[0.92 ; 1.49]$

$1.18[0.93 ; 1.50]$

$1.15[0.90 ; 1.47]$

$1.15[0.90 ; 1.47]$

$1.17[0.91 ; 1.49]$

$1.18[0.93 ; 1.49]$

$1.17[0.92 ; 1.49]$

$1.13[0.89 ; 1.44]$

$1.16[0.91 ; 1.48]$

$1.16[0.91 ; 1.48]$

$1.13[0.88 ; 1.45]$

$1.14[0.89 ; 1.46]$

$1.15[0.90 ; 1.47]$

$1.12[0.87 ; 1.43]$

$1.12[0.91 ; 1.37]$

$1.10[0.86 ; 1.41]$

$1.16[0.91 ; 1.48]$

$1.10[0.86 ; 1.40]$

$1.15[0.90 ; 1.46]$

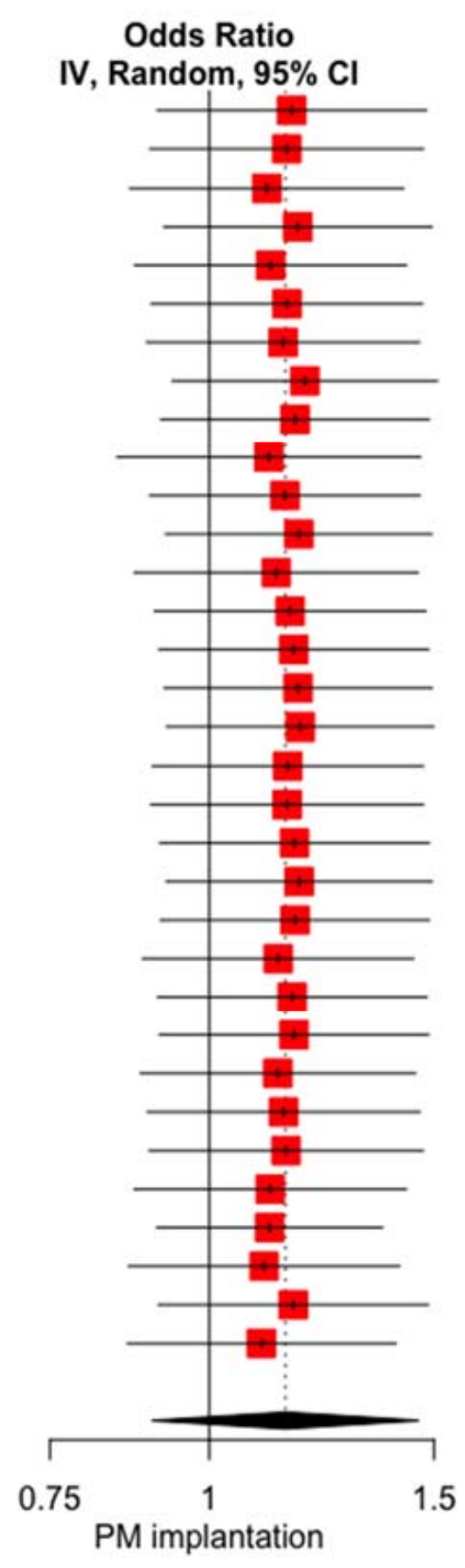

Odds Ratio

Figure 2. Forest plot leave one-out analysis comparing the effect of the presence of LBBB on the rate of post-TAVI PPI. $\mathrm{IV}=$ interval variable; $95 \% \mathrm{CI}=$ confidence interval.

\subsection{Influence of RBBB on PPI}

Data on RBBB were extracted from 28 studies (19-46); among the 46,663 patients included in the analysis, there were 31,603 showing pre-implant RBBB. The cumulative proportion of RBBB was 9.2\% (7.3\%-11.6\%) (Supplementary Figure S9). Heterogeneity was high $(97.8 \%$ [97.3\%; 98.1\%]). No publication bias was found $(p=0.1112)$. The cumulative proportion of RBBB in a subset of 6932 patients with post-TAVI PPI was $24.7 \%$ $(19.6 \%-30.6 \%)$ with high heterogeneity $(94.6 \%$ [93.2\%; $95.8 \%]$ ) and no publication bias $(p=0.1023)$ (Supplementary Figure S10). The cumulative proportion of RBBB in a subset of 39,670 patients without post-TAVI PPI was $6.3 \%(4.9 \%-8.1 \%)$ with high heterogeneity $\left(\mathrm{I}^{2} 96.6 \%[95.9 \% ; 97.3 \%]\right)$ and no publication bias $(p=0.2659)$ (Supplementary Figure S11). 
The influence of RBBB on post-TAVI PPI was significant, with OR $4.8581(4.1571 ; 5.6775)$, $p<0.0001$ with moderate heterogeneity $\mathrm{I}^{2}=63.4 \%[45.1 \% ; 75.6 \%]$ and no publication bias $(p=0.937)$ (Figure 3). The baujat plot (Supplementary Figure S12) shows that two studies $[36,37]$ may impact the heterogeneity. Sensitivity analysis confirms the impact of RBBB on the post-TAVI PPI rate even at the leave-one out analysis (Figure 4). Meta-regression failed to identify some modifiers: age $(\mathrm{r}=-0.0592 ; p=0.4292)$, left ventricular ejection fraction (LVEF, $\mathrm{r}=0.0246, p=0.3755)$, and year of the study $(\mathrm{r}=0.0489, p=0.3038)$ did not show any influence on meta-analytic results (Supplementary Figures S13-S15).

\begin{tabular}{|c|c|c|c|c|c|c|}
\hline \multirow[b]{2}{*}{ Study } & \multicolumn{2}{|c|}{ Experimental } & \multicolumn{3}{|c|}{ Control } & \multirow{2}{*}{$\begin{array}{c}\text { Odds Ratio } \\
\text { IV, Random, } \mathbf{9 5 \%} \mathrm{Cl}\end{array}$} \\
\hline & Events & Total & Events & Total & Weight & \\
\hline De Carlo 2012 & 15 & 32 & 51 & 243 & $2.7 \%$ & $3.311[1.564 ; 7.006]$ \\
\hline Gensas 2014 & 22 & 41 & 67 & 311 & $3.1 \%$ & $4.179[2.152 ; 8.116]$ \\
\hline Mouillet 2015 & 60 & 115 & 192 & 718 & $4.8 \%$ & $2.981[1.999 ; 4.448]$ \\
\hline Zif 2015 & 82 & 312 & 91 & 1661 & $5.3 \%$ & $6.143[4.425 ; 8.529]$ \\
\hline Rodriguez-Olivares 2016 & 13 & 28 & 55 & 274 & $2.5 \%$ & $3.445[1.568 ; 7.566]$ \\
\hline Watabe 2016 & 18 & 108 & 19 & 647 & $3.0 \%$ & $6.589[3.361 ; 12.917]$ \\
\hline Gonska 2017 & 13 & 22 & 39 & 261 & $2.1 \%$ & $8.005[3.266 ; 19.616]$ \\
\hline Raelson 2017 & 19 & 65 & 35 & 513 & $3.3 \%$ & $5.652[3.013 ; 10.604]$ \\
\hline Dumonteuil 2017 & 20 & 26 & 61 & 224 & $2.0 \%$ & $8.385[3.309 ; 21.244]$ \\
\hline Monteiro 2017 & 36 & 71 & 99 & 599 & $4.0 \%$ & $5.172[3.107 ; 8.610]$ \\
\hline Auffret 2017 & 137 & 362 & 370 & 3165 & $6.0 \%$ & $4.601[3.626 ; 5.838]$ \\
\hline Pellegrini 2018 & 6 & 22 & 22 & 261 & $1.8 \%$ & $4.193[1.534 ; 11.459]$ \\
\hline De-Torres-Alba 2018 & 7 & 20 & 69 & 586 & $2.0 \%$ & $4.137[1.637 ; 10.454]$ \\
\hline Mangieri 2018 & 7 & 37 & 47 & 574 & $2.3 \%$ & $2.731[1.165 ; 6.403]$ \\
\hline Bhardwaj 2018 & 11 & 50 & 33 & 333 & $2.7 \%$ & $2.612[1.237 ; 5.515]$ \\
\hline Pellegrini 2018 & 30 & 63 & 85 & 646 & $3.8 \%$ & $5.979[3.482 ; 10.268]$ \\
\hline Gaede 2018 & 31 & 98 & 145 & 927 & $4.4 \%$ & $2.510[1.587 ; 3.969]$ \\
\hline deem 2018 & 65 & 113 & 81 & 559 & $4.5 \%$ & 7.929 [5.110; 12.303] \\
\hline Chamandi 2018 & 84 & 169 & 238 & 1460 & $5.3 \%$ & $5.066[3.638 ; 7.054]$ \\
\hline Doshi 2018 & 96 & 220 & 1853 & 7928 & $5.8 \%$ & $2.541[1.938 ; 3.331]$ \\
\hline Vejpongsa 2018 & 265 & 715 & 1560 & 17685 & $6.5 \%$ & $6.090[5.188 ; 7.149]$ \\
\hline Cresse 2019 & 6 & 14 & 20 & 372 & $1.5 \%$ & $13.149[4.316 ; 40.063]$ \\
\hline Dolci 2019 & 12 & 29 & 23 & 237 & $2.3 \%$ & $6.520[2.810 ; 15.126]$ \\
\hline Maeno 2019 & 38 & 101 & 66 & 558 & $4.2 \%$ & $4.490[2.792 ; 7.222]$ \\
\hline Costa 2019 & 39 & 92 & 106 & 1024 & $4.4 \%$ & $6.368[4.030 ; 10.062]$ \\
\hline Meduri 2019 & 68 & 85 & 177 & 619 & $3.7 \%$ & $9.758[5.613 ; 16.966]$ \\
\hline Du 2020 & 6 & 20 & 32 & 236 & $1.8 \%$ & $2.821[1.041 ; 7.640]$ \\
\hline Shivamurthy 2020 & 38 & 130 & 52 & 787 & $4.3 \%$ & $5.831[3.649 ; 9.318]$ \\
\hline otal $(95 \% \mathrm{Cl})$ & & 3160 & & 43408 & $100.0 \%$ & $4.858[4.157 ; 5.677]$ \\
\hline eterogeneity: $\mathrm{T}$ & & 3.74 & & ( 0.01): & $=63 \%$ & \\
\hline
\end{tabular}

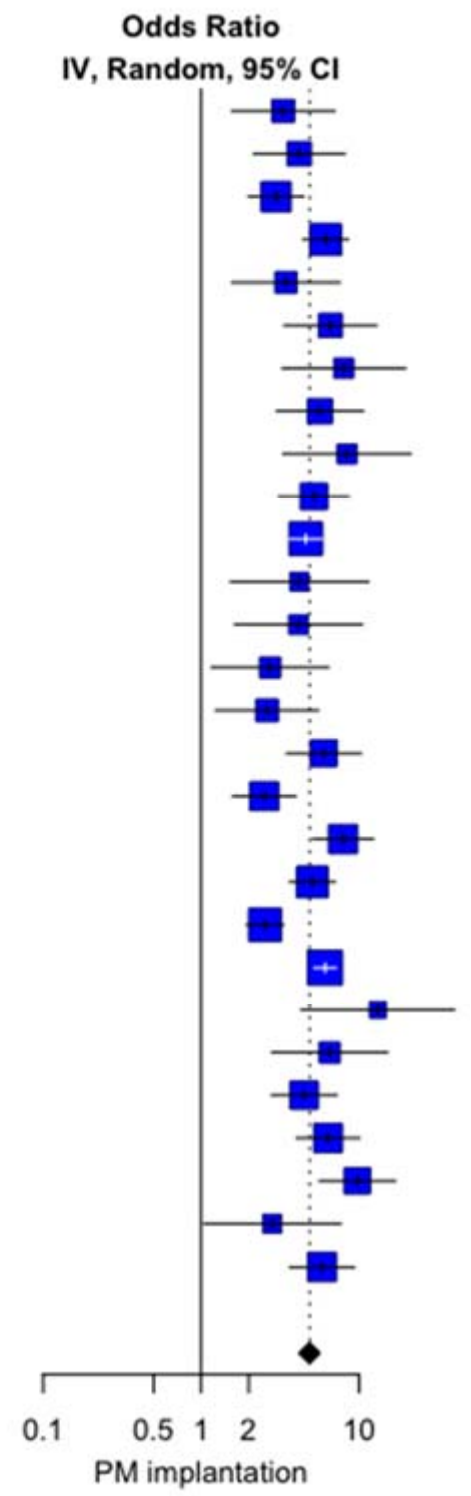

Figure 3. Forest plot comparing the effect of RBBB on the rate of post-TAVI PPI. 
post-TAVI PPI.

IV = interval variable; $95 \% \mathrm{CI}=$ confidence interval.

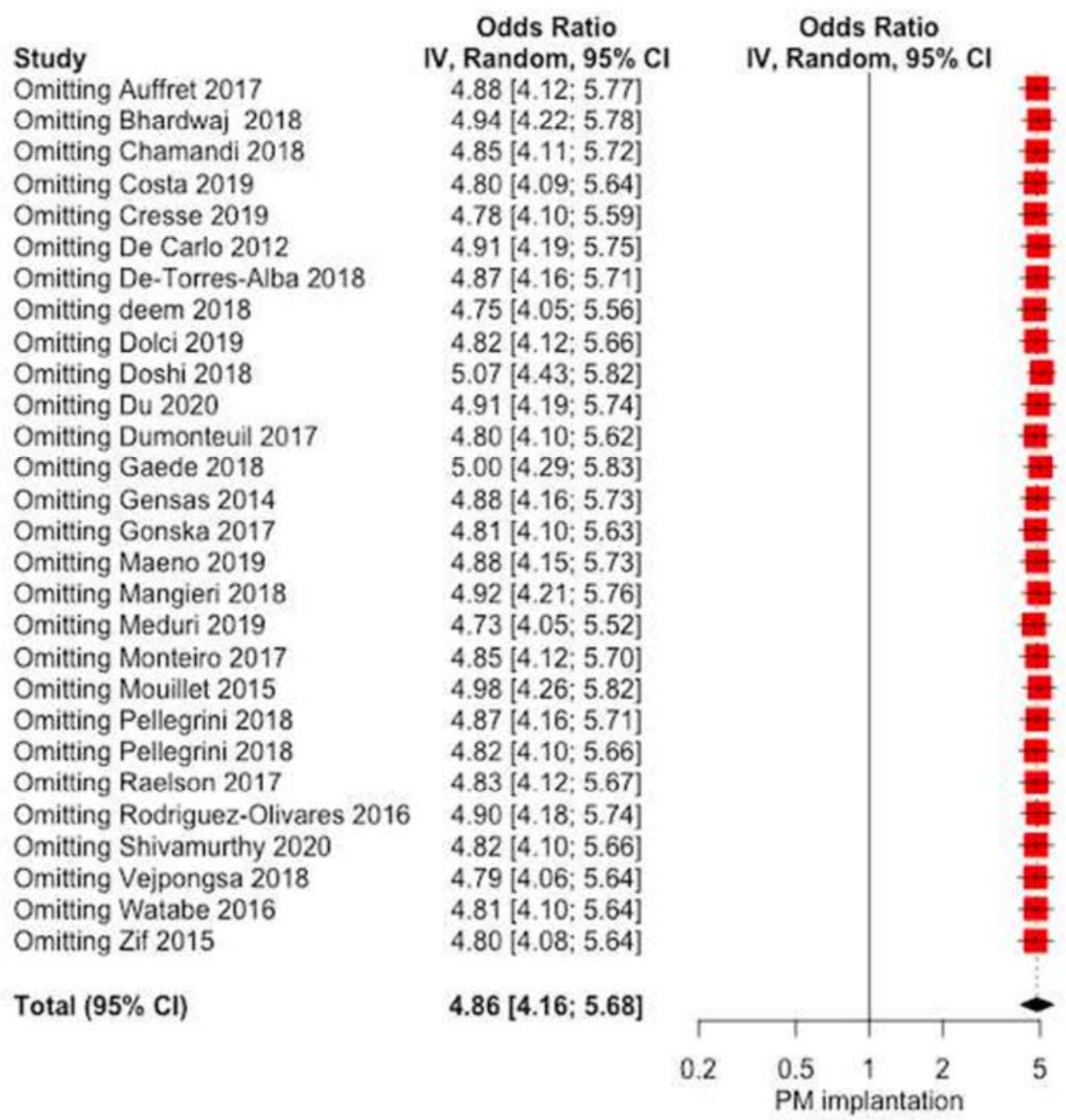

Figure 4. Forest plot leave one-out analysis comparing the effect of the presence of RBBB on the rate of post-TAVI PPI.

\section{Discussion}

Our meta-analysis demonstrates the impact of bundle branch block on post-TAVI PPI. Our study is derived from 36 studies reporting clinical outcomes in 55,851 patients receiving TAVI and presenting bundle branch conduction disturbances. The main results of this study can be assumed as follows: (i) the presence of LBBB does not influence post-TAVI PPI; (ii) the presence of RBBB has a significant impact on post-TAVI PPI.

The prevalence of LBBB in TAVI candidates is closed to $10 \%$ according to previous studies $[45,55,56]$ and new-onset LBBB occurs in $\cong 30 \%$ after TAVI, depending on the type of prosthesis [9,57]. In the present study, the cumulative proportion of LBBB was $11.9 \%$, including pre-operative LBBB and new-onset LBBB after TAVI. This rate is thus in accordance with previously published data $[4,58]$. The impact of LBBB on post-TAVI PPI remains controversial $[49,50,59]$. Data from the PARTNER experience $[50,53]$ suggested that new-onset LBBB increases the rate of post-TAVI PPI. Moreover, an analysis at a national registry level as the prospective open TAVI Karlsruhe registry [48] identified slightly more PPI in patients with persistent new-onset LBBB. However, current data do not promote prophylactic PPI in patients presenting new-onset LBBB after TAVI [60] and 
some studies failed to identify LBBB as predictors for post-TAVI PPI [58,61]. In our study, the influence of LBBB on post-TAVI PPI was not significant. These controversial results can be related to various definitions of LBBB according to the degree of QRS prolongation defining the LBBB $[58,61-63]$ or to the high heterogeneity among the current studies [64]. Moreover, only three studies $[22,50,53]$ quantified LBBB as left anterior fascicular or left posterior fascicular block, which may underestimate the impact of LBBB on post-TAVI PPI. As development of high-degree atrio-ventricular block is shown to be a common complication in patients with pre-existing LBBB (or RBBB) after TAVI [65], we shared the view of Waksman and colleagues [66] emphasizing the need for routine electrophysiology evaluation in patients undergoing TAVI in order to limit post-TAVI PPI. Electrophysiology testing differentiates the supra-nodal conduction disturbances from the infra-nodal ones by analyzing the atrial-His and the His-ventricular intervals. By integrating these values into the baseline electrocardiogram, we may be able to predict the evolution of the atrioventricular conduction in high-grade atrio-ventricular block. Such data may help the clinicians to choose between an aggressive strategy of early post-TAVI PPI or, on the contrary, an expectative strategy, avoiding some unnecessary post-TAVI PPI. The results of current ongoing trials investigating the use of electrophysiology-based algorithmic approaches with HV measurements as a guide for PPI, particularly in patients with LBBB (Clinical Monitoring Strategy vs. EP-Guided Algorithmic in LBBB Patients Post-TAVR (NCT03303612); The MARE Study (NCT02153307); Prospective Validation of a Pre-Specified algorithm for the management of conduction disturbances following transcatheter aortic valve replacement PROMOTE (NCT04139616)), are expected with impatience.

Pre-existing RBBB has been demonstrated in 6.9\% [67] up to $13.6 \%$ [44] of patients undergoing TAVI and is a common underlying conduction disturbance in TAVI patients [10]. In this study, the cumulative proportion of RBBB among the included studies reached $9.2 \%$, also in accordance with the studies of Auffret and colleagues [45], reporting a prevalence of RBBB of $10.3 \%$ in TAVI candidates. Several studies have already highlighted the role of pre-existing RBBB in the development of atrioventricular conduction, leading to post-TAVI PPI $[2,3,22,26,33,39,41]$. Our findings confirm the impact of such a condition on post-TAVI PPI, without the influence of age or LVEF. As previous studies emphasized the higher all-cause mortality and the poorer clinical outcomes in patients with pre-existing RBBB $[10,45]$, bradyarrhythmia events may participate in the poorer outcomes of these patients. However, this analysis does not provide the cause of such discrepancies between RBBB and LBBB. The fragility of the right bundle branch during minor trauma or procedures may partially explain its highest incidence in the general population and, consequently, the more important impact of RBBB in post-TAVI PPI [68]. We look forward to having definitive data and guidelines to determine the optimal management and monitoring of patients with RBBB undergoing TAVI [60].

The current guidelines with respect to post-TAVI PPI [69] do not specify the adequate timing for post-TAVI PPI. Previously, the 2013 European Society Guidelines [70] recommended a clinical rhythm observation period of 7 days in the presence of high-grade trio-ventricular block before proceeding to post-TAVI PPI. In the current meta-analysis, the range of timing for PPI varied from day 0 to 1 year, also emphasizing the need for PPI after discharge and the influence of atrio-ventricular conduction in the long term [49]. This hypothetical observation period may be shortened or lengthened according to patient-related factors and electrical findings. Once again, electrophysiological study may identify some high-risk patients for the development of high-grade atrio-ventricular disturbances and, thereby, adjust the observation rhythm period in such patients [71].

As the definitive impact of infra-Hisian conduction disturbances on post-TAVI PPI has not definitively been clarified yet, specific recommendations for the type of valves used in such patients must be done with caution. However, some studies investigated the impact of the valves used on post-TAVI conduction disturbances $[3,44,49,61]$. Indeed, Franzoni and colleagues [61] found a higher incidence of LBBB when using a Medtronic CoreRevalving System with respect to the Edwards Sapien Valve. A more intra-ventricular implantation 
of the stent valve frame may result in higher rate of post-TAVI PPI [49]. Furthermore, the gap between the lower edge of the coronary cusp and the end of the frame of the prosthetics valve has been shown to be greater in patients with new-onset LBBB than in patients with post-operative conduction disturbances [61], emphasizing the role of the prosthesis placement in the development of post-operative conduction disturbances. Nevertheless, the requirement for post-TAVI PPI is multifactorial and studies incorporating electrophysiological findings with procedural data are mandatory [72].

\section{Study Limitations}

This meta-analysis has several limitations to be acknowledged. First, this metaanalysis possesses all the inherent bias associated with this investigation technique. Second, including pre-operative LBBB and new-onset LBBB in the analysis may be considered as a limitation for the interpretation of the final results, as the pre-operative LBBB can be related to other cardiovascular conditions and the new-onset LBBB can be related to procedural/interventional aspects. However, as our primary outcome was 30-day or inhospital PPI, we can afford to compare both LBBB as conduction disturbances present after TAVI, which can lead to PPI, independently of the chronic or acute apparition of the LBBB. Third, distinction of LBBB in left anterior fascicular or left posterior fascicular block was lacking in the majority of the included studies, thereby not providing enough information to perform in-depth analysis. Further research in this respect is currently needed. Finally, analysis of individual patient-level data may provide further understandings.

\section{Conclusions}

This meta-analysis found that patients with the presence of RBBB have higher risk for post-TAVI PPI, with no influence of age or LVEF. This finding has not been confirmed for patients experimenting with LBBB. Pre-operative evaluation strategies, including electrophysiological characteristics, are crucial in further extending patient selection for TAVI.

Supplementary Materials: The following are available online at https:/ /www.mdpi.com/article/10 $.3390 / \mathrm{jcm} 10122719 / \mathrm{s} 1$, Figure S1. Flowsheet of the included studies, Figure S2. Forest plot pooling the proportion of LBBB in 33 studies, Figure S3. Forest plot pooling the proportion of LBBB in subset of 7315 patients with post-TAVI PPI. Figure S4. Forest plot pooling the proportion of LBBB in subset of 43,650 patients without post-TAVI PPI, Figure S5. Baujat plot: impact of the studies on overall heterogeneity in studies reporting on LBBB status, Figure S6. Bubble plots: influence of age on risk for post-TAVI PPI in patients with LBBB, Figure S7. Bubble plots: influence of LVEF on risk for post-TAVI PPI in patients with LBBB, Figure S8. Bubble plots: influence of the year of the study on risk for post-TAVI PPI in patients with LBBB, Figure S9. Forest plot pooling the proportion of RBBB in 28 studies, Figure S10. Forest plot pooling the proportion of RBBB in subset of 6932 patients with post-TAVI PPI, Figure S11. Forest plot pooling the proportion of RBBB in subset of 39,670 patients without post-TAVI PPI, Figure S12. Baujat plot: impact of the studies on overall heterogeneity in studies reporting on RBBB status, Figure S13. Bubble plots: influence of age on risk for post-TAVI PPI in patients with RBBB, Figure S14. Bubble plots: influence of LVEF on risk for post-TAVI PPI in patients with RBBB, Figure S15. Bubble plots: influence of the year of the study on risk for post-TAVI PPI in patients with RBBB.

Author Contributions: Conceptualization, J.M.R., M.D.M. and R.L.; methodology, J.M.R., M.D.M. and R.L.; software, M.D.M.; validation, K.V., S.M., A.W.V.H., S.K. and L.V.; formal analysis, J.M.R. and M.D.M.; investigation, J.M.R. and M.D.M.; resources, S.M., D.R. and J.S.; data curation, J.M.R. and M.D.M.; writing — original draft preparation, J.M.R. and M.D.M.; writing — review and editing, J.M.R., M.D.M. and R.L.; visualization, J.M.R., M.D.M., K.V., S.M., D.R., J.S., A.W.V.H., L.V., S.K., J.G.M. and R.L.; supervision, S.K. and R.L.; project administration, J.M.R., M.D.M. and R.L.; funding acquisition, none. All authors have read and agreed to the published version of the manuscript.

Funding: This research received no external funding. 
Institutional Review Board Statement: Because this study was a systematic review and metaanalysis based on published articles, ethical approval was waived by the institutional review board of the University Hospital of Maastricht.

Informed Consent Statement: Patient consent was waived due to the systematic review and metaanalytic nature of this study.

Data Availability Statement: No new data were created or analyzed in this study. Data sharing is not applicable to this article.

Conflicts of Interest: The authors declare no conflict of interest.

\section{References}

1. Erkapic, D.; De Rosa, S.; Kelava, A.; Lehmann, R.; Fichtlscherer, S.; Hohnloser, S.H. Risk for permanent pacemaker after transcatheter aortic valve implantation: A comprehensive analysis of the literature. J. Cardiovasc. Electrophysiol. 2012, 23, 391-397. [CrossRef]

2. Siontis, G.C.; Jüni, P.; Pilgrim, T.; Stortecky, S.; Büllesfeld, L.; Meier, B.; Wenaweser, P.; Windecker, S. Predictors of permanent pacemaker implantation in patients with severe aortic stenosis undergoing TAVR: A meta-analysis. J. Am. Coll. Cardiol. 2014, 64, 129-140. [CrossRef] [PubMed]

3. Van Gils, L.; Tchetche, D.; Lhermusier, T.; Abawi, M.; Dumonteuil, N.; Rodriguez-Olivares, R.; Molina-Martin, D.N.J.; Stella, P.R.; Carrié, D.; De Jaegere, P.P.; et al. Transcatheter Heart Valve Selection and Permanent Pacemaker Implantation in Patients with Pre-Existent Right Bundle Branch Block. J. Am. Heart Assoc. 2017, 6, e005028. [CrossRef]

4. $\quad$ Lopez-Aguilera, J.; Saint-Gerons, J.M.S.; Bellido, F.M.; Suarez, D.L.H.D.T.J.; Pineda, S.O.; Alvarez-Ossorio, M.P.; Angel, R.M.M.; Pavlovic, D.; Suarez, D.L.C.C.J. Effect of New-Onset Left Bundle Branch Block After Transcatheter Aortic Valve Implantation (CoreValve) on Mortality, Frequency of Re-Hospitalization, and Need for Pacemaker. Am. J. Cardiol. 2016, 118, 1380-1385. [CrossRef]

5. Rodès-Cabau, J. Transcatheter aortic valve implantation: Current and future approaches. Nat. Rev. Cardiol. 2012, 9, 15-29. [CrossRef] [PubMed]

6. Waksman, R.; Rogers, T.; Torguson, R.; Gordon, P.; Ehsan, A.; Wilson, S.R.; Goncalves, J.; Levitt, R.; Hahn, C.; Parikh, P.; et al. Transcatheter Aortic Valve Replacement in Low-Risk Patients with Symptomatic Severe Aortic Stenosis. J. Am. Coll. Cardiol. 2018, 72, 2095-2105. [CrossRef] [PubMed]

7. Finkelstein, A.; Rozenbaum, Z.; Halkin, A.; Banai, S.; Bazan, S.; Barbash, I.; Segev, A.; Fefer, P.; Maor, E.; Danenberg, H.; et al. Outcomes of Transcatheter Aortic Valve Implantation in Patients with Low Versus Intermediate to High Surgical Risk. Am. J. Cardiol. 2019, 123, 644-649. [CrossRef]

8. Voigtländer, L.; Seiffert, M. Expanding TAVI to Low and Intermediate Risk Patients. Front. Cardiovasc. Med. 2018, 5, 92. [CrossRef] [PubMed]

9. Regueiro, A.; Abdul-Jawad, A.O.; Del Trigo, M.; Campelo-Parada, F.; Puri, R.; Urena, M.; Philippon, F.; Rodès-Cabau, J. Impact of New-Onset Left Bundle Branch Block and Periprocedural Permanent Pacemaker Implantation on Clinical Outcomes in Patients Undergoing Transcatheter Aortic Valve Replacement: A systematic Review and Meta-Analysis. Circ. Cardiovasc. Interv. 2016, 9, e003635. [CrossRef] [PubMed]

10. Croix, G.R.S.; Spencer, C.; Hrachian, H.L.; Beohar, N. Clinical Impact of Preexisting Right Bundle Branch Block after Transcatheter Aortic Valve Replacement: A Systematic Review and Meta-Analysis. J. Interv. Cardiol. 2020, 2020, 1789516.

11. Massouillé, G.; Bordachar, P.; Ellenbogen, K.A.; Souteyrand, G.; Jean, F.; Combaret, N.; Vorilhon, C.; Clerfond, G.; Farhat, M.; Ritter, P.; et al. New-onset left bundle branch block induced by transcutaneous aortic valve implantation. Am. J. Cardiol. 2016, 117, 867-873. [CrossRef] [PubMed]

12. Rivard, L.; Schram, G.; Asgar, A.; Khairy, P.; Andrade, J.G.; Bonan, R.; Dubuc, M.; Guerra, P.G.; Ibrahim, R.; Macle, L.; et al. Electrocardiographic and electrophysiological predictors of atrioventricular block after transcatheter aortic valve replacement. Heart Rhythm. 2015, 12, 321-329. [CrossRef] [PubMed]

13. Warton, D.I.; Hui, F.K.C. The arcsine is asinine: The analysis of proportions in ecology. Ecology 2011, 92, 3-10. [CrossRef] [PubMed]

14. DerSimonian, R.; Laird, N. Meta-analysis in clinical trials revisited. Contemp. Clin. Trials 2015, 45 Pt A, 139-145. [CrossRef]

15. Bowden, J.; Tierney, J.F.; Copas, A.J.; Burdett, S. Quantifying, displaying and accounting for heterogeneity in the meta-analysis of RCTs using standard and generalized Q statistics. BMC Med. Res. Methodol. 2011, 11, 41-52. [CrossRef]

16. Huedo-Medina, T.B.; Sanchez-Meca, J.; Marin-Martinez, F.; Botella, J. Assessing heterogeneity in meta-analysis: Q statistic or I2 index? Psychol. Methods 2006, 11, 193-206. [CrossRef] [PubMed]

17. Begg, C.B.; Mazumdar, M. Operating characteristics of a rank correlation test for publication bias. Biometrics 1994, 50, $1088-1101$. [CrossRef] [PubMed]

18. Baujat, B.; Mahé, C.; Pignon, J.-P.; Hill, C. A graphical method for exploring heterogeneity in meta-analyses: Application to a meta-analysis of 65 trials. Stat. Med. 2002, 21, 2641-2652. [CrossRef] [PubMed] 
19. De Carlo, M.; Giannini, C.; Bedogni, F.; Klugmann, S.; Brambilla, N.; De Marco, F.; Zuchelli, G.; Testa, L.; Oreglia, J.; Petronio, A.S. Safety of a conservative strategy of permanent pacemaker implantation after transcatheter aortic CoreValve implantation. Am. Heart J. 2012, 163, 492-499. [CrossRef] [PubMed]

20. Gensas, C.S.; Caixeta, A.; Siqueira, D.; Carvalho, L.A.; Sarmento-Leite, R.; Mangione, J.A.; Lemos, P.A.; Colafranceschi, A.S.; Caramori, P.; Ferreira, M.C.; et al. Brazilian Registry in Transcatheter Aortic Valve Implantation Investigators. Predictors of permanent pacemaker requirement after transcatheter aortic valve implantation: Insights from a Brazilian registry. Int. J. Cardiol. 2014, 175, 248-252. [CrossRef]

21. Mouillet, G.; Lellouche, N.; Yamamoto, M.; Oguri, A.; Dubois-Rande, J.L.; Van Belle, E.; Gilard, M.; Laskar, M.; Teiger, E. Outcomes following pacemaker implantation after transcatheter aortic valve implantation with CoreValve $\left({ }^{\circledR}\right)$ devices: Results from the FRANCE 2 Registry. Catheter. Cardiovasc. Interv. 2015, 86, E158-E166. [CrossRef] [PubMed]

22. Nazif, T.M.; Dizon, J.M.; Hahn, R.T.; Xu, K.; Babaliaros, V.; Douglas, P.S.; El-Chami, M.F.; Herrmann, H.C.; Mack, M.; Makkar, R.R.; et al. Predictors and clinical outcomes of permanent pacemaker implantation after transcatheter aortic valve replacement: The PARTNER (Placement of AoRtic TraNscathertER Valves) trial and registry. JACC Cardiovasc. Interv. 2015, 8 Pt A, 60-69. [CrossRef]

23. Rodríguez-Olivaresa, R.; van Gils, L.; El Faquir, N.; Rahhab, Z.; Di Martino, L.F.M.; van Weenen, S.; de Vriesa, J.; Galema, T.W.; Geleijnse, M.L.; Budde, R.P.; et al. Importance of the left ventricular outflow tract in the need for pacemaker implantation after transcatheter aortic valve replacement. Int. J. Cardiol. 2016, 216, 9-15. [CrossRef]

24. Gonska, B.; Seeger, J.; Keßler, M.; Von Keil, A.; Rottbauer, W.; Wöhrle, J. Predictors for permanent pacemaker implantation in patients undergoing transfemoral aortic valve implantation with the Edwards Sapien 3 valve. Clin. Res. Cardiol. 2017, 106, 590-597. [CrossRef] [PubMed]

25. Raelson, C.A.; Gabriels, J.; Ruan, J.; Ip, J.E.; Thomas, G.; Liu, C.F.; Cheung, J.W.; Lerman, B.B.; Patel, A.; Markowitz, S.M. Recovery of atrioventricular conduction in patients with heart block after transcatheter aortic valve replacement. J. Cardiovasc. Electrophysiol. 2017, 28, 1196-1202. [CrossRef]

26. Dumonteil, N.; Meredith, I.T.; Blackman, D.J.; Tchétché, D.; Hildick-Smith, D.; Spence, M.S.; Walters, D.L.; Harnek, J.; Worthley, S.G.; Rioufol, G.; et al. Insights into the need for permanent pacemaker following implantation of the repositionable LOTUS valve for transcatheter aortic valve replacement in 250 patients: Results from the REPRISE II trial with extended cohort. Euro Interv. 2017, 13, 796-803. [CrossRef]

27. Monteiro, C.; Di Leoni, F.A.; Caramori, P.R.; Carvalho, L.A.F.; Siqueira, D.A.; Sao, T.L.E.K.; Perin, M.; De Lima, V.C.; Guérios, E.; Brito, F.S.D., Jr.; et al. Permanent Pacing After Transcatheter Aortic Valve Implantation: Incidence, Predictors and Evolution of Left Ventricular Function. Arq. Bras. Cardiol. 2017, 109, 550-559. [CrossRef] [PubMed]

28. Pellegrini, C.; Kim, W.K.; Holzamer, A.; Walther, T.; Mayr, N.P.; Michel, J.; Rheude, T.; Nuñez, J.; Kasel, A.M.; Trenkwalder, T.; et al. Multicenter Evaluation of Prosthesis Oversizing of the SAPIEN 3 Transcatheter Heart Valve Impact on Device Failure and New Pacemaker Implantations. Rev. Esp. Cardiol. 2019, 72, 641-648. [CrossRef] [PubMed]

29. De-Torres-Alba, F.; Kaleschke, G.; Vormbrock, J.; Orwat, S.; Radke, R.; Feurle, M.; Diller, G.P.; Reinecke, H.; Baumgartner, H. Delayed pacemaker requirement after transcatheter aortic valve implantation with a new-generation balloon expandable valve: Should we monitor longer? Int. J. Cardiol. 2018, 273, 56-62. [CrossRef] [PubMed]

30. Mangieri, A.; Lanzillo, G.; Bertoldi, L.; Jabbour, R.J.; Regazzoli, D.; Ancona, M.B.; Tanaka, A.; Mitomo, S.; Garducci, S.; Montalto, C.; et al. Predictors of Advanced Conduction Disturbances Requiring a Late ( $\geq 48 \mathrm{~h})$ Permanent Pacemaker Following Transcatheter Aortic Valve Replacement. JACC Cardiovasc. Interv. 2018, 11, 1519-1526. [CrossRef]

31. Bhardwaj, A.; Ramanan, T.; Sawant, A.C.; Sinibaldi, E.; Pham, M.; Khan, S.; Qureshi, R.; Agrawal, N.; Khalil, C.; Hansen, R.; et al. Quality of life outcomes in transcatheter aortic valve replacement patients requiring pacemaker implantation. J. Arrhythm. 2018, 34, 441-449. [CrossRef] [PubMed]

32. Pellegrini, C.; Husser, O.; Kim, W.K.; Holzamer, A.; Walther, T.; Rheude, T.; Mayr, N.P.; Trenkwalder, T.; Joner, M.; Michel, J.; et al. Predictors of Need for Permanent Pacemaker Implantation and Conduction Abnormalities With a Novel Self-expanding Transcatheter Heart Valve. Rev. Esp. Cardiol. 2019, 72, 145-153. [CrossRef]

33. Gaede, L.; Kim, W.K.; Liebetrau, C.; Dörr, O.; Sperzel, J.; Blumenstein, J.; Berkowitsch, A.; Walther, T.; Hamm, C.; Elsässer, A.; et al. Pacemaker implantation after TAVI: Predictors of AV block persistence. Clin. Res. Cardiol. 2018, 107, 60-69. [CrossRef] [PubMed]

34. Nadeem, F.; Tsushima, T.; Ladas, T.P.; Thomas, R.B.; Patel, S.M.; Saric, P.; Patel, T.; Lipinski, J.; Li, J.; Costa, M.A.; et al. Impact of Right Ventricular Pacing in Patients Who Underwent Implantation of Permanent Pacemaker After Transcatheter Aortic Valve Implantation. Am. J. Cardiol. 2018, 122, 1712-1717. [CrossRef] [PubMed]

35. Chamandi, C.; Barbanti, M.; Munoz-Garcia, A.; Latib, A.; Nombela-Franco, L.; Gutiérrez-Ibanez, E.; Veiga-Fernandez, G.; Cheema, A.N.; Cruz-Gonzalez, I.; Serra, V.; et al. Long-Term Outcomes in Patients with New Permanent Pacemaker Implantation Following Transcatheter Aortic Valve Replacement. JACC Cardiovasc. Interv. 2018, 11, 301-310. [CrossRef] [PubMed]

36. Doshi, R.; Decter, D.H.; Meraj, P. Incidence of arrhythmias and impact of permanent pacemaker implantation in hospitalizations with transcatheter aortic valve replacement. Clin. Cardiol. 2018, 41, 640-645. [CrossRef]

37. Vejpongsa, P.; Zhang, X.; Bhise, V.; Kitkungvan, D.; Shivamurthy, P.; Anderson, H.V.; Balan, P.; Nguyen, T.C.; Estrera, A.L.; Dougherty, A.H.; et al. Risk Prediction Model for Permanent Pacemaker Implantation after Transcatheter Aortic Valve Replacement. Struct. Heart 2018, 2, 328-335. [CrossRef] 
38. Cresse, S.; Eisenberg, T.; Alfonso, C.; Cohen, M.G.; DeMarchena, E.; Williams, D.; Carrillo, R. Cardiac conduction abnormalities associated with pacemaker implantation after transcatheter aortic valve replacement. Pacing Clin. Electrophysiol. 2019, 42, 846-852. [CrossRef] [PubMed]

39. Dolci, G.; Vollema, E.M.; Van Der Kley, F.; De Weger, A.; Marsa, N.A.; Delgado, V.; Bax, J.J. One-Year Follow-Up of Conduction Abdormalities After Transcatheter Aortic Valve Implantation with the SAPIEN 3 Valve. Am. J. Cardiol. 2019, 124, $1239-1245$. [CrossRef] [PubMed]

40. Costa, G.; Zappulla, P.; Barbanti, M.; Cirasa, A.; Todaro, D.; Rapisarda, G.; Picci, A.; Platania, F.; Tosto, A.; Di Grazia, A.; et al. Pacemaker dependency after transcatheter aortic valve implantation: Incidence, predictors and long-term outcomes. EuroIntervention 2019, 15, 875-883. [CrossRef]

41. Meduri, C.U.; Kereiakes, D.J.; Rajagopal, V.; Makkar, R.R.; O’Hair, D.; Linke, A.; Waksman, R.; Babliaros, V.; Stoler, R.C.; Mishkel, G.J.; et al. Pacemaker Implantation and Dependency After Transcatheter Aortic Valve Replacement in the REPRISE III Trial. J. Am. Heart Assoc. 2019, 8, e012594. [CrossRef] [PubMed]

42. Du, F.; Zhu, Q.; Jiang, J.; Chen, H.; Liu, X.; Wang, J. Incidence and Predictors of Permanent Pacemaker Implantation in Patients Who Underwent Transcatheter Aortic Valve Replacement: Observation of a Chinese Population. Cardioliology 2019, 145, 27-34. [CrossRef] [PubMed]

43. Shivamurthy, P.; Vejpongsa, P.; Gurung, S.; Jacob, R.; Zhao, Y.; Anderson, H.V.; Balan, P.; Nguyen, T.C.; Estrera, A.L.; Dougherty, A.H.; et al. Validation of Scoring System Predicting Permanent Pacemaker Implantation after Transcatheter Aortic Valve Replacement. Pacing Clin. Electrophysiol. 2020, 43, 479-485. [CrossRef] [PubMed]

44. Watanabe, Y.; Kozuma, K.; Hioki, H.; Kawashima, H.; Nara, Y.; Kataoka, A.; Nagura, F.; Nakashima, M.; Shirai, S.; Tada, N.; et al. Pre-Existing Right Bundle Branch Block Increases Risk for Death After Transcatheter Aortic Valve Replacement with a Balloon-Expandable Valve. JACC Cardiovasc. Interv. 2016, 9, 2210-2216. [CrossRef] [PubMed]

45. Auffret, V.; Webb, J.G.; Eltchaninoff, H.; Munoz-Garcia, A.J.; Himbert, D.; Tamburino, C.; Nombela-Franco, L.; Nietlispach, F.; Moris, C.; Ruel, M.; et al. Clinical Impact of Baseline Right Bundle Branch Block in Patients Undergoing Transcatheter Aortic Valve Replacement. JACC Cardiovasc. Interv. 2017, 10, 1564-1574. [CrossRef]

46. Maeno, Y.; Abramowitz, Y.; Israr, S.; Yoon, S.-H.; Kubo, S.; Nomura, T.; Miyasaka, H.; Kawamori, H.; Kazuno, Y.; Takahashi, N.; et al. Prognostic impact of Permanent Pacemaker Implantation in Patients with Low Left Ventricular Ejection Fraction Following Transcatheter Aortic Valve Replacement. J. Invasive Cardiol. 2019, 31, E15-E22.

47. Testa, L.; Latib, A.; De Marco, F.; De Carlo, M.; Agnifili, M.; Latino, R.A.; Petronio, A.S.; Ettori, F.; Poli, A.; De Servi, S.; et al. Clinical Impact of persistent left bundle-branch block after transcatheter aortic valve implantation with CoreValve Revalving System. Circulation 2013, 127, 1300-1307. [CrossRef] [PubMed]

48. Schymik, G.; Tzamalis, P.; Bramlage, P.; Heimeshoff, M.; Würth, A.; Wondraschek, R.; Gonska, B.-D.; Posival, H.; Schmitt, C.; Schröfel, H.; et al. Clinical impact of a new left bundle branch block following TAVI implantation: 1-year results of the TAVIK cohort. Clin. Res. Cardiol. 2014, 104, 351-362. [CrossRef] [PubMed]

49. Urena, M.; Webb, J.G.; Cheema, A.; Serra, V.; Toggweiler, S.; Barbanti, M.; Cheung, A.; Ye, J.; Dumont, E.; DeLarochellière, R.; et al. Impact of New-Onset Persistent Left Bundle Branch Block on Late Clinical Outcomes in Patients Undergoing Transcatheter Aortic Valve Implantation with a Balloon-Expandable Valve. JACC Cardiovasc. Interv. 2014, 7, 128-136. [CrossRef] [PubMed]

50. Nazif, T.M.; Williams, M.R.; Hahn, R.T.; Kapadia, S.; Babaliaros, V.; Rodés-Cabau, J.; Szeto, W.Y.; Jilaihawi, H.; Fearon, W.F.; Dvir, D.; et al. Clinical implications of new-onset left bundle branch block after transcatheter aortic valve replacement: Analysis of the PARTNER experience. Eur. Heart J. 2014, 35, 1599-1607. [CrossRef] [PubMed]

51. Fischer, Q.; Himbert, D.; Webb, J.G.; Eltchaninoff, H.; Munoz-Garcia, A.J.; Tamburino, C.; Nombela-Franco, L.; Nietlispach, F.; Moris, C.; Ruel, M.; et al. Impact of Preexisting Left Bundle Branch Block in Transcatheter Aortic Valve Replacement Recipients. Circ. Cardiovasc. Interv. 2018, 11, e006927. [CrossRef] [PubMed]

52. Chamandi, C.; Barbanti, M.; Munoz-Garcia, A.; Latib, A.; Nombela-Franco, L.; Guttiérrez-Ibanez, E.; Veiga-Fernandez, G.; Cheema, A.N.; Cruz-Gonzales, I.; Serra, V.; et al. Long-term Outcomes in Patients with New-Onset Persistent Left Bundle Branch Block Following TAVR. JACC Cardiovasc. Interv. 2019, 12, 1175-1184. [CrossRef] [PubMed]

53. Nazif, T.M.; Chen, S.; George, I.; Dizon, J.M.; Hahn, R.T.; Crowley, A.; Alu, M.C.; Babaliaros, V.; Thourani, V.H.; Herrmann, H.C.; et al. New-onset left bundle branch block after transcatheter aortic valve replacement is associated with adverse long-term clinical outcomes in intermediate-risk patients: An analysis from the PARTNER II trial. Eur. Heart J. 2019, 40, 2218-2227. [CrossRef] [PubMed]

54. Hamandi, M.; Tabachnick, D.; Lanfear, A.T.; Baxter, R.; Shin, K.; Zingler, B.; Mack, M.J.; DiMaio, J.M.; Kindsvater, S. Effect of new and persistent left bundle branch block after transcatheter aortic valve replacement on long-term need for pacemaker implantation. Bayl. Univ. Med. Cent. Proc. 2020, 33, 157-162. [CrossRef]

55. Urena, M.; Webb, J.G.; Eltchaninoff, H.; Munoz-Garcia, A.J.; Bouleti, C.; Tamburino, C.; Nombela-Franco, L.; Nietlispach, F.; Moris, C.; Ruel, M.; et al. Late cardiac death in patients undergoing transcatheter aortic valve replacement: Incidence and predictors of advanced heart failure and sudden cardiac death. J. Am. Coll. Cardiol. 2015, 65, 437-448. [CrossRef] [PubMed]

56. Erkapic, D.; Kim, W.-K.; Weber, M.; Möllmann, H.; Berkowitsch, A.; Zaltsberg, S.; Pajitnev, D.J.; Rixe, J.; Neumann, T.; Kuniss, M.; et al. Electrocardiographic and further predictors for permanent pacemaker requirement after transcatheter aortic valve implantation. Europace 2010, 12, 1188-1190. [CrossRef] [PubMed] 
57. Van der Boon, R.M.; Nuis, R.J.; Van Mieghem, N.M.; Jordaens, L.; Rodès-Cabau, J.; Van Domburg, R.T.; Seruys, P.W.; Anderson, R.H.; De Jaegere, P.P.T. New conduction abnormalities after TAVI-frequency and causes. Nat. Rev. Cardiol. 2012, 9, 454-463. [CrossRef]

58. Sasaki, K.; Izumo, M.; Kuwata, S.; Ishibashi, Y.; Kamijima, R.; Watanabe, M.; Kaihara, T.; Okuyama, K.; Koga, M.; Nishikawa, H.; et al. Clinical Impact of New-Onset Left Bundle-Branch Block After Transcatheter Aortic Valve Implantation in the Japanese Population. Circ. J. 2020, 84, 1012-1019. [CrossRef] [PubMed]

59. Keßler, M.; Gonska, B.; Seeger, J.; Rottbauer, W.; Wöhrle, J. Long-term clinical outcome of persistent left bundle branch block after transfemoral aortic valve implantation. Catheter. Cardiovasc. Interv. 2018, 93, 538-544. [CrossRef] [PubMed]

60. Muntané-Carol, G.; Guimaraes, L.; Ferreira-Neto, A.N.; Wintzer-Wehekind, J.; Junquera, L.; Del Val, D.; Faroux, L.; Philippon, F.; Rodés-Cabau, J. How does new-onset left bundle branch block affect the outcomes of transcatheter aortic valve repair? Expert Rev. Med. Devices 2019, 16, 589-602. [CrossRef]

61. Franzoni, I.; Latib, A.; Maisano, F.; Costopoulos, C.; Testa, L.; Figini, F.; Giannini, F.; Basavarajaiah, S.; Mussardo, M.; Slavich, M.; et al. Comparison of Incidence and Predictors of Left Bundle Branch Block After Transcatheter Aortic Valve Implantation Using the CoreValve Versus the Edwards Valve. Am. J. Cardiol. 2013, 112, 554-559. [CrossRef]

62. Urena, M.; Mok, M.; Serra, V.; Dumont, E.; Nombela-Franco, L.; DeLarochellière, R.; Doyle, D.; Igual, A.; Larose, E.; Amat-Santos, I.; et al. Predictive factors and long-term clinical consequences of persistent left bundle branch block following transcatheter aortic valve implantation with a balloon expandable valve. J. Am. Coll. Cardiol. 2012, 60, 1743-1752. [CrossRef]

63. Vernooy, K.; Verbeek, X.A.; Peschar, M.; Crijns, H.J.G.M.; Arts, T.; Cornelussen, R.N.M.; Prinzen, F.W. Left bundle branch block induces ventricular remodeling and functional septal hypoperfusion. Eur. Heart J. 2005, 26, 91-98. [CrossRef] [PubMed]

64. Shoar, S.; Batra, S.; Gulraiz, I.W.; Javed, M.; Hosseini, F.; Naderan, M.; Shoar, N.; John, J.; Modukuru, V.R.; Sharma, S.K. Effect of pre-existing left bundle branch block on post-procedural outcomes of transcatheter aortic valve replacement: A meta-analysis of comparative studies. Am. J. Cardiovasc. Dis. 2020, 10, 294-300. [PubMed]

65. Egger, F.; Nürnberg, M.; Rohla, M.; Weiss, T.W.; Unger, G.; Smetana, P.; Geppert, A.; Gruber, S.C.; Bambazek, A.; Falkensammer, J.; et al. High-degree atrioventricular block in patients with preexisting bundle branch block or bundle branch block occurring during transcatheter aortic valve implantation. Heart Rhythm. 2014, 11, 2176-2182. [CrossRef] [PubMed]

66. Waksman, R.; Khan, J.M. Left Bundle Branch Block After TAVR. Buddle or Trouble? JACC Cardiovasc. Interv. 2019, 12, 1185-1187. [CrossRef] [PubMed]

67. Husser, O.; Pellegrini, C.; Kim, W.-K.; Holzamer, A.; Pilgrim, T.; Toggweiler, S.; Schäfer, U.; Blumenstein, J.; Deuschl, F.; Rheude, T.; et al. Transcatheter Valve SELECTion in Patients with Right Bundle Branch Block and Impact on Pacemaker Implantation J. Am. Coll. Cardiol. Interv. 2019, 12, 1781-1793. [CrossRef] [PubMed]

68. Kumpuris, A.G.; Casale, T.B.; Mokotoff, D.M.; Miller, R.R.; Luchi, R.J. Right bundle-branch block. Occurrence following nonpenetrating chest trauma without evidence of cardiac contusion. JAMA 1979, 242, 172-173. [CrossRef] [PubMed]

69. Kusumoto, F.M.; Schoenfeld, M.H.; Barrett, C.; Edgerton, J.R.; Ellenbogen, K.A.; Gold, M.R.; Goldschlager, N.F.; Hamilton, R.M.; Joglar, J.A.; Kim, R.J.; et al. 2018 ACC/AHA/HRS Guideline on the Evaluation and Management of Patients with Bradycardia and Cardiac Conduction Delay: A Report of the American College of Cardiology/American Heart Association Task Force on Clinical Practice Guidelines and the Heart Rhythm Society. Circulation 2019, 140, e382-e482. [PubMed]

70. Brignole, M.; Auricchio, A.; Baron-Esquivias, G.; Bordachar, P.; Boriani, G.; Breithardt, O.-A.; Cleland, J.; Deharo, J.-C.; Delgado, V.; Elliott, P.M.; et al. 2013 ESC Guidelines on cardiac pacing and cardiac resynchronization therapy. Eur. Heart J. 2013, 34, $2281-2329$. [CrossRef] [PubMed]

71. Knecht, S.; Schaer, B.; Reichlin, T.; Spies, F.; Madaffari, A.; Vischer, A.; Fahrni, G.; Jeger, R.; Kaiser, C.; Osswald, S.; et al. Electrophysiology Testing to Stratify Patients with Left Bundle Branch Block After Transcatheter Aortic Valve Implantation. J. Am. Heart Assoc. 2020, 9, e014446. [CrossRef] [PubMed]

72. Sandhu, A.; Tzou, W.; Ream, K.; Valle, J.; Tompkins, C.; Nguyen, D.T.; Sauer, J.D.; Messenger, J.; Aleong, R.G. Heart Block After Discharge in Patients Undergoing TAVR with Latest-Generation Valves. J. Am. Coll. Cardiol. 2018, 71, 577-578. [CrossRef] [PubMed] 